\title{
Equações utilizadas para a predição de frequência cardíaca máxima na população pediátrica não atlética: uma revisão sistemática
}

\section{Equations used to predict maximum heart rate in the non-athletic pediatric population:} a systematic review

\author{
Andressa Schenkel Spitznagel (D) 1, Dyovana Silva dos Santos (D) 1, Karolini Reis Branco (D) 1, Daniele Schiwe (D) 2, \\ Vinícius da Silva Lessa de Oliveira (D) ${ }^{1}$, João Paulo Heinzmann-Filho (D) ${ }^{1,2} \square$ \\ ' Curso de Fisioterapia, Centro Universitário Cenecista de Osório (UNICNEC). Osório, Rio Grande do Sul, Brasil. \\ 2 Programa de Pós-graduação em Pediatria e Saúde da Criança, Pontifícia Universidade Católica do Rio Grande do Sul (PUCRS). Rio Grande do Sul, Brasil.
}

Como citar este artigo (How to cite this article):

Spitznagel AS, Santos DS, Branco KR, Schiwe D, Oliveira VSL, Heinzmann-Filho JP. Equações utilizadas para a predição de frequência cardíaca máxima na população pediátrica não atlética: uma revisão sistemática (Equations used to predict maximum heart rate in the non-athletic pediatric population: a systematic review). Sci Med.29(4):e34286. https://doi.org/10.15448/1980-6108.2019.4.34286

\section{RESUMO}

OBJETIVOS: Revisar a aplicabilidade das equações preditivas de frequência cardíaca máxima durante os testes de exercício em crianças e adolescentes não atletas.

MÉTODOS: Trata-se de uma revisão sistemática, realizada no Pubmed, Lilacs, Scielo e PEDro. Foram incluídos estudos que compararam a frequência cardíaca máxima medida e a estimada por equações preditivas durante testes de esforço em crianças e adolescentes não atletas. Utilizou-se a seguinte estratégia de pesquisa: Exercise test OR Exercise testing OR Cardiopulmonary exercise test OR Cardiopulmonary exercise testing OR Peak oxygen uptake OR Maximal oxygen consumption OR Exercise tolerance OR Exercise capacity AND Heart rate OR Heart rates OR Pulse rate OR Pulse rates OR Heart rate control OR Cardiac chronotropic OR Predictive value test AND Predictive equations. A qualidade metodológica foi avaliada pela escala da Agency for Health Care Research and Quality.

RESULTADOS: De um total de 858 artigos localizados, apenas quatro foram incluídos. Os artigos totalizaram 325 participantes (sete a 18 anos). Todos os estudos mensuraram a frequência cardíaca máxima por meio do teste de esforço cardiopulmonar. Enquanto nenhum estudo $(04 / 04=100 \% ; 01 / 01=100 \%)$ recomendou as fórmulas " 220 - idade" e a "207 - 0,7 x idade", as equações "208 - (0,7 x idade)" e a " 200 - 0,48 $\mathrm{x}$ idade" foram adequadas em $02 / 03(66,6 \%)$ artigos e $01 / 01(100 \%)$ documentos, respectivamente. A qualidade metodológica foi considerada alta em todos os artigos avaliados, oscilando entre 76 e 97 pontos.

CONCLUSÕES: Os achados parecem sugerir que a fórmula "208 - (0,7 x idade)" foi a equação mais testada e adequada em grande parte para estimar os valores de frequência cardíaca máxima em crianças e adolescentes não atletas. No entanto, ainda são necessários mais estudos para confirmar esses resultados.

DESCRITORES: Frequência cardíaca, equações preditivas, teste de exercício, pediatria.

\section{ABSTRACT}

AIMS: To evaluate the applicability of the predictive equations of maximum heart rate during exercise tests in non-athlete children and adolescents.

METHODS: It is a systematic review, carried out through Pubmed, Lilacs, Scielo and PEDro. We included studies comparing the maximum heart rate measured and estimated by predictive equations during stress tests in non-athlete children and adolescents. The following search strategy was used: Exercise test OR Exercise testing OR Cardiopulmonary exercise test OR Cardiopulmonary exercise testing OR Peak oxygen uptake OR Maximal oxygen consumption OR Exercise tolerance OR Exercise capacity AND Heart rate OR Heart rates OR Pulse rate OR Pulse rates OR Heart rate control OR Cardiac chronotropic OR Predictive value test AND Predictive equations. The methodological quality was assessed by the Agency for Health Care Research and Quality scale.

RESULTS: Of a total of 858 articles located, only four were included. The articles totaled 325 participants (seven to 18 years). All studies measured the maximum heart rate by cardiopulmonary stress test. While no study recommended $(04 / 04=100 \% ; 01 / 01=100 \%)$ the formulas "220 - age" and "207 - $0.7 \mathrm{x}$ age", the equations "208 - (0.7 x age)" and "200 - $0.48 \mathrm{x}$ age" were adequate in 02/03 (66.6\%) articles and 01/01 $(100 \%)$ document, respectively. The methodological quality was considered high in all articles evaluated, ranging from 76 to 97 points.

CONCLUSIONS: The findings seem to suggest that the formula "208- $(0.7 \mathrm{x}$ age $)$ " was the most tested and adequate equation to a large extent for estimating maximum heart rate in non-athlete children and adolescents. However, further studies are still needed to confirm these results. KEYWORDS: Heart rate, predictive equations, exercise test, pediatrics. 
Abreviaturas: FC, frequência cardíaca; FCmáx, frequência cardíaca máxima.

\section{INTRODUÇÃO}

A frequência cardíaca (FC) é considerada uma das variáveis fisiológicas mais importantes, podendo ser definida pelo número de vezes que a bomba cardíaca contrai dentro de um minuto. Essa variável aumenta linearmente de acordo com a progressão do exercício físico, sendo a frequência cardíaca máxima (FCmáx) o número máximo de batimentos que o coração realiza em um minuto [1].

O sistema nervoso autônomo é o responsável pela regulação da FC. O período de repouso ocorre devido à dominância do sistema nervoso autônomo parassimpático, atuando sobre o nodo sinoatrial, que é responsável pelo ritmo das batidas cardíacas. Quando o indivíduo realiza alguma atividade física, o sistema nervoso autônomo simpático predomina, ocorrendo a elevação fisiológica do número de batimentos cardíacos [2].

Diversas causas podem ser responsáveis por alterar a FC na população pediátrica, incluindo cardiopatias, prematuridade e patologias neurológicas $[3,4]$. Isso pode levar a redução da capacidade de consumo máximo de oxigênio, podendo desencadear dispneia compelida pelo exercício, impactando negativamente na sua qualidade de vida $[5,6]$.

$\mathrm{Na}$ prática clínica, a FC é frequentemente utilizada devido a sua fácil mensuração e também como um bom indicador de morbidade. Quando elevada pode estar associada a riscos relacionados ao sistema cardiovascular, como doenças coronarianas, por exemplo [7]. Além disso, é um método que permite prescrever a intensidade de exercícios de forma adequada, utilizando-se geralmente uma faixa em torno de 60 a $85 \%$ da FCmáx. Também, é comumente utilizada como um critério de interrupção do teste de esforço físico, exigindo-se valores acima de $85 \%$ do previsto [8].

Ainda, a FC de repouso costuma ser menor em atletas praticantes de atividades aeróbias, em relação a indivíduos sedentários, tendo como hipóteses (I) de que em indivíduos atletas ocorre uma elevação de estímulos do sistema nervoso autônomo parassimpático sobre o nódulo sinoatrial, aumentando o tônus vagal, e (II) de que a FC mais baixa é consequência de um mecanismo intrínseco existente no nódulo sinoatrial [9].

A verificação da FCmáx, pode ser mensurada diretamente através de testes máximos (cicloergômetro ou esteira) ou indiretamente por meio de equações preditivas [10]. Nos protocolos envolvendo ergômetros, realiza-se exercícios de forma gradual e sem interrupções, elevando-se a $\mathrm{FC}$ até um valor previamente calculado [11]. Outra forma de estimar a FCmáx é através de equações preditivas, em que se supõe o valor através de cálculos que envolvem a FC de repouso, idade, e outras variáveis, conforme as particularidades de cada equação [12].

Atualmente, existem diversas fórmulas para a predição da FCmáx na literatura, sendo as equações de Karvonen et al. e de Tanaka et al. as mais investigadas e utilizadas em laboratórios de fisiologia do exercício [12-17]. Karvonen et al., em 1957 atribuiu a equação de FCmáx baseada na subtração de " 220 " da idade cronológica do avaliado, sendo recomendada pela American College of Sports Medicine. Além disso, outra equação foi proposta por Tanaka et al. em 2001 , em que se subtrai o valor de " 208 " da idade do participante, corrigido por " 0,7 " [18].

Embora as equações preditivas de FCmáx sejam amplamente utilizadas na população adulta, na amostra pediátrica pode subestimar ou superestimar os valores de FC, podendo predizer de maneira inadequada a variável em questão [19]. O viés na estimativa da FCmáx pode dificultar a melhora da aptidão física, o fortalecimento da bomba cardíaca e não exigir um gasto cardiovascular ideal para um sujeito jovem, justificando o desenvolvimento deste estudo [20]. Assim, o objetivo do trabalho foi revisar a aplicabilidade das equações preditivas de FCmáx durante os testes em exercícios de crianças e adolescentes não atletas.

\section{MÉTODOS}

Trata-se de uma revisão de literatura, do tipo sistemática. Foram incluídos estudos observacionais, em que compararam os resultados de FCmáx obtida nos testes máximos com os valores estimados através de equações preditivas em crianças e adolescentes ( $<18$ anos) não atletas (definido por não praticar nenhum exercício físico regular). Os estudos que realizaram testes submáximos, pesquisas que incluíram amostras de atletas/não atletas (não estratificada), artigos de revisão de literatura, opiniões de especialistas e cartas ao revisor foram excluídos.

Os bancos de dados utilizados na pesquisa foram o Pubmed/Medline, Lilacs, Scielo e PEDro. A busca utilizada para a seleção dos artigos foi baseada nas seguintes palavras-chave, associados com descritores booleanos: Exercise test OR Exercise testing OR Cardiopulmonary exercise test OR Cardiopulmonary 
exercise testing OR Peak oxygen uptake OR Maximal oxygen consumption OR Exercise tolerance OR Exercise capacity AND Heart rate OR Heart rates OR Pulse rate OR Pulse rates OR Heart rate control OR Cardiac chronotropic OR Predictive value test AND Predictive equations. Esses deveriam constar nos títulos, resumos e/ou nas palavras-chave. Também foram utilizadas as referências dos artigos incluídos em nossa revisão para selecionar possíveis pesquisas relacionadas ao tema (grey literature).

Dois avaliadores (A.S.S. e D.S.S.) realizaram de forma independente a pesquisa dos artigos, visto que, quando ocorreu discordância entre os avaliadores, a mesma foi resolvida por consenso com um terceiro avaliador (J.P.H.F.). Posteriormente, quando identificados os descritores, realizou-se a leitura dos mesmos para verificar se os artigos estavam de acordo com os critérios de inclusão e exclusão.

Não foram utilizados filtros quanto ao idioma e ao período de publicação dos artigos. As buscas foram realizadas no mês de agosto a outubro de 2018. Para a extração dos dados, registraram-se as seguintes informações: sobrenome do primeiro autor, ano de publicação, país da coleta de dados, número da amostra, faixa etária, grupos avaliados, teste realizado, tipo de protocolo, equações preditivas testadas, valor medido e estimado de FCmáx e os principais resultados.

Para a avaliação da qualidade metodológicas dos artigos, utilizou-se a escala da Agency for Health Care Research and Quality. A escala avalia nove itens relacionados à questão do estudo, aspectos metodológicos, coerência dos resultados, discussão e patrocínio. Quando somado cada item, o resultado pode totalizar até 100 pontos. Os artigos são classificados conforme a pontuação obtida, sendo essa categorizada em baixa ( $<50$ pontos), moderada (50 até 66 pontos) e alta qualidade metodológica ( $>66$ pontos) [21].

\section{RESULTADOS}

Nas buscas realizadas nas bases de dados, foram encontrados 858 artigos, sendo 734 localizados no
Pubmed, 95 no Scielo, 29 no Lilacs e nenhum artigo no PEDro. Desses, 70 foram excluídos por estarem repetidos nas bases de dados e 786 pelo fato de não preencherem os critérios de elegibilidade. Assim, selecionou-se o total de dois estudos através das bases de dados e dois artigos por meio da grey literature, resultando em um total de quatro documentos (Figura 1).

Os artigos totalizaram 325 participantes, sendo compostos por 59 obesos e 266 sem a avaliação do índice de massa corporal. As amostras variaram de 52 até 76 sujeitos, com a faixa etária, oscilando entre sete e 18 anos. Os indivíduos eram predominantemente do sexo masculino. Para a avaliação da FCmáx, todos os estudos utilizaram o teste de esforço cardiopulmonar, sendo que três (75\%) dos quatro artigos utilizaram o protocolo de rampa e um (25\%) utilizou o protocolo de Bruce adaptado. A Tabela 1 apresenta as informações amostrais de cada estudo.
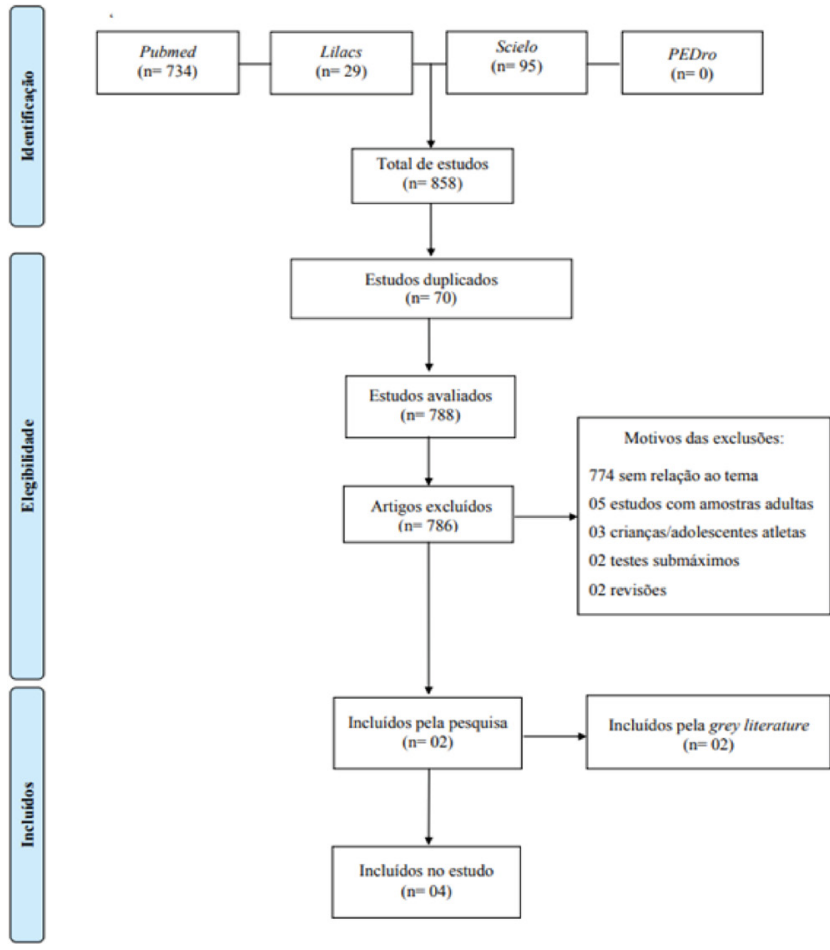

Figura 1. Sistematização das pesquisas incluídas no trabalho.

Tabela 1. Características dos estudos incluídos nessa revisão.

\begin{tabular}{|c|c|c|c|c|}
\hline Autoria & Origem & Idade (anos) & $\mathbf{n}$ & Grupos analisados \\
\hline Mahon et al., 2010 [23] & Estados Unidos & $7-17$ & 52 & Não atletas \\
\hline Machado e Denadai, 2011 [1] & Brasil & $10-16$ & 69 & Não atletas \\
\hline Colantonio e Kiss, 2013 [22] & Brasil & $7-17$ & $\begin{array}{l}69 \\
76\end{array}$ & $\begin{array}{l}\text { Sujeitos treinados* } \\
\text { Não atletas }\end{array}$ \\
\hline Heinzmann-Filho et al., 2018 [16] & Brasil & $15-18$ & 59 & Não atletas obesos \\
\hline
\end{tabular}

* Nadadores; n: Tamanho amostral. 
Todos os artigos utilizaram a equação "220idade"; três $(75 \%)$ deles testaram a equação "208$(0,7 \times$ idade $) " ;$ um a fórmula "207-0,7 $\times$ idade" e uma pesquisa a equação " $200-0,48 \times$ idade" para as estimativas de FCmáx. Nenhum estudo recomendou o uso das fórmulas "220 - idade" e a "207-0,7×idade", superestimando os valores previstos de FCmáx nas amostras pediátricas. Além disso, duas $(66,6 \%)$ e uma $(100 \%)$ indicaram estimativas adequadas da FCmáx pela equação " 208 - $(0,7 \times$ idade $)$ " e da " 200 $0,48 \times$ idade", respectivamente (Tabela 2 ).

Por fim, todas as pesquisas apresentaram alta qualidade metodológica, obtendo uma pontuação média de 84,5 pontos, oscilando entre 76 e 97 . Os estudos perderam pontuação nos itens sobre população do estudo, comparabilidade dos indivíduos, análise estatística, resultados, financiamento e patrocínios. Alguns itens dessa escala não se aplicam ao delineamento em questão, sendo assim, os artigos acabaram pontuando, considerando que não seria adequado retirar a pontuação deles. Dentre esses, destacam-se a comparabilidade das características amostrais entre os grupos, a comparação entre os participantes e aqueles que não foram alocados no trabalho e também quanto ao tempo adequado de seguimento. A Tabela 3 apresenta a pontuação em cada domínio avaliado conforme a escala da Agency for Health Care Research and Quality.

Tabela 2. Principais resultados dos estudos incluídos.

\begin{tabular}{|c|c|c|c|}
\hline Autor \& ano de publicação & Equações preditivas & FCmáx (bpm) & Resultados da FCmáx \\
\hline \multirow[t]{3}{*}{ Mahon et al., 2010 [23] } & & Mensurada: $201 \pm 10,0$ & \\
\hline & 220-idade & Prevista: $208 \pm 3,0$ & Fórmula supertima \\
\hline & $208-(0,7 x$ idade $)$ & Prevista: $200 \pm 2,0$ & Fórmula estima adequadamente \\
\hline \multirow[t]{3}{*}{ Machado e Denadai, 2011 [1] } & & Mensurada: $200,2 \pm 8,0$ & \\
\hline & 220 - idade & Prevista: $207,4 \pm 1,5$ & Fórmula supertima \\
\hline & 208 - (0,7 xidade $)$ & Prevista: $199,2 \pm 1,1$ & Fórmula estima adequadamente \\
\hline \multirow[t]{2}{*}{ Colantonio e Kiss, 2013 [22] } & & Mensurada: $182,29 \pm 15,52 *$ & \\
\hline & 220-idade & Prevista: $\Delta$ FCmáx 23,85† & Fórmula supertima \\
\hline \multirow[t]{5}{*}{ Heinzmann-Filho et al., 2018 [16] } & & Mensurada: $190 \pm 9,2$ & \\
\hline & 220-idade & Prevista: $203,2 \pm 1,2$ & Fórmula supertima \\
\hline & $208-(0,7 \times$ idade $)$ & Prevista: $196,3 \pm 0,8$ & Fórmula supertima \\
\hline & $207-(0,7 \times$ idade $)$ & Prevista: $195,3 \pm 0,8$ & Fórmula supertima \\
\hline & $200-(0,48 \times$ idade $)$ & Prevista: $191,9 \pm 0,6$ & Fórmula estima adequadamente \\
\hline
\end{tabular}

FCmáx: Frequência cardíaca máxima.

* Dados da FCmáx das meninas do grupo não atleta (11-14 anos); ${ }^{\dagger}$ Artigo relata a diferença da FCmáx (valor previsto - valor medido).

Tabela 3. Pontuação referente à qualidade metodológica dos artigos.

\begin{tabular}{|c|c|c|c|c|c|}
\hline Critérios avaliados & $\begin{array}{l}\text { Pontuação de } \\
\text { referência }\end{array}$ & $\begin{array}{c}\text { Mahon et al., } \\
2010 \text { [23] }\end{array}$ & $\begin{array}{c}\text { Machado e Denadai, } \\
2011 \text { [1] }\end{array}$ & $\begin{array}{l}\text { Colantonio Kiss, } \\
2013[22]\end{array}$ & $\begin{array}{l}\text { Heinzmann-Filho et al., } \\
2018[16]\end{array}$ \\
\hline Pergunta do estudo & 2 & 2 & 2 & 2 & 2 \\
\hline População do estudo & 8 & 5 & 5 & 5 & 8 \\
\hline $\begin{array}{l}\text { Comparabilidade dos } \\
\text { indivíduos para os estudos } \\
\text { observacionais }\end{array}$ & 22 & 17 & 17 & 17 & 19 \\
\hline Exposição ou intervenção & 11 & 11 & 11 & 11 & 11 \\
\hline Medidas de resultados & 20 & 20 & 20 & 20 & 20 \\
\hline Análise estatística & 19 & 12 & 12 & 10 & 19 \\
\hline Resultados & 8 & 8 & 8 & 6 & 8 \\
\hline Discussão & 5 & 5 & 5 & 5 & 5 \\
\hline Financiamento e patrocínio & 5 & 0 & 5 & 0 & 5 \\
\hline Escore total & 100 & 80 & 85 & 76 & 97 \\
\hline
\end{tabular}




\section{DISCUSSÃO}

A FCmáx é frequentemente utilizada como um marcador para a prescrição de exercícios aeróbicos em amostras adultas e pediátricas. Embora na população adulta parece que a equação " 220 - idade" é adequada para estimar os resultados da FCmáx, na população pediátrica não atlética essa estimativa pode causar erros nas predições, baseada em nossa revisão [22]. Dois estudos mostraram que a equação " $208-(0,7 \times$ idade $)$ " foi a fórmula mais testada e adequada em grande parte para predizer os resultados da FCmáx, apesar de outra pesquisa ter superestimado esses resultados $[1,16,23]$.

O cálculo " $208-(0,7 \times$ idade $)$ " foi criado por Tanaka et al., no ano de 2001 através de uma metanálise envolvendo 351 estudos. A revisão incluiu 18.712 indivíduos adultos saudáveis (predomínio do sexo masculino), tanto composto por atletas, bem como, por sedentários, sem a utilização de medicamentos. A partir disso, foi gerado um modelo regressão de linear, a fins de prever a FCmáx nessa amostra. Assim, obteve-se a criação da equação "208-(0,7×idade)" [24]. Em nossa revisão, dois estudos relataram que a equação "208$(0,7 \times$ idade $)$ " foi adequada para estimar os resultados de FCmáx em crianças e adolescentes não atletas. Esses estudos avaliaram amostras com idade entre 7 e 17 anos, sendo compostas por não atletas e com predomínio do sexo masculino [1,23]. Tal fato poderia explicar, pelo menos em parte, a estimativa adequada dessa equação, já que houve um predomínio de homens também no estudo de Tanaka et al. 2001. Além disso, já é esperado que sujeitos do gênero masculino costumam atingir FC mais elevadas, quando comparadas ao sexo feminino. Já outro estudo, relatou que a equação "208(0,7×idade)" superestimou os achados de FCmáx [16]. Esses achados podem ser relacionados aos participantes serem em grande maioria do sexo feminino e com sobrepeso ou obesidade, diferenciando-se das amostras dos estudos anteriores $[1,23]$.

Do ponto de vista da fisiologia do exercício, os próprios testes de esforço podem gerar resultados considerados aceitáveis nas suas mensurações, oscilando em até 12 batimentos por minuto [1]. Dessa forma, o estudo de Heinzmann-Filho et al. acabou superestimando 13,2 $\pm 1,2$ batimentos por minuto com o uso da fórmula "208 - (0,7 x idade)", ultrapassando os valores considerados aceitáveis dessas medições. Embora ainda não seja o ideal, utilizar uma única equação para predizer o desempenho cardiovascular seria o mais prático. No entanto, parece que para cada amostra, grupo etário, tipo de esporte praticado e outros fatores como o nível de atividade física (ativo ou inativo), torna-se necessário uma reflexão sobre qual a melhor equação preditiva. Uma forma mais prática, mas que deve ser utilizada com cautela, é utilização de pontos de coorte para as predições de FCmáx em amostras jovens. Gelbart et al. realizaram um estudo transversal para determinar um ponto de corte para FCmáx de crianças e adolescentes (atletas e não atletas). A média de FCmáx encontrada foi de 197 batimentos por minuto, sugerindo-se o ponto de corte de 180 batimentos por minuto, considerando que $97,2 \%$ dos indivíduos analisados obtiveram valores acima desse resultado [10].

A equação "220 - idade" se mostrou inadequada para a população pediátrica não atleta, superestimando a FCmáx nesse grupo. Embora essa fórmula seja recomendada pela American College of Sports Medicine, parece que em amostras jovens atléticas e geriátricas, os valores são superestimados e subestimados, respectivamente [25]. Frequentemente esse cálculo é atribuído a Åstrand, Karvonen et al. e alguns outros autores, porém, sua autoria é incerta. Essa equação se desenvolveu através da observação de alguns estudos que não foram publicados. Ainda, estudos indicam que a equação foi realizada com adultos portadores de doenças crônicas, diferindo assim, de amostras pediátricas saudáveis [26]. Dessa forma, não se recomenda o uso da fórmula preditiva de "220-idade" para a amostra pediátrica atlética e não atlética, baseado em nossa revisão [27].

O cálculo "207-0,7×idade" não se mostrou adequado, apresentando resultados superestimados. Essa fórmula foi gerada através de uma análise retrospectiva de mensurações de FCmáx, incluindo 4.666 indivíduos, de ambos sexos. Isso pode justificarse pelo fato de que a equação foi baseada somente em amostras adultas (27 até 78 anos), utilizando um protocolo com aumentos de velocidade/inclinação associados, o que poderia levar a um estresse cardiovascular excessivo, atingindo valores maiores de FCmáx [28]. Além disso, apenas o estudo de HeinzmannFilho et al. testou a equação "200-0,48×idade" em amostras pediátricas não atléticas, com o estado nutricional elevado (sobrepeso/obesidade) [16]. A equação "200-0,48 × idade" estimou adequadamente a FCmáx de crianças obesas, o que poderia ser atribuído ao fato de que a equação proposta também foi oriunda de amostras com obesidade. No entanto, um dos motivos para não generalizarmos o uso dessa equação para toda faixa etária pediátrica não atlética se deve pelo motivo de apenas 1 estudo ter avaliado a aplicação dessa equação preditiva, bem como, por incluir sujeitos com índice de massa corporal elevado, o que poderia 
não apresentar o mesmo poder preditivo para sujeitos eutróficos [16].

Todos os artigos incluídos em nossa revisão utilizaram o teste de esforço cardiopulmonar para mensurar a FCmáx. Classicamente, os testes de esforço são utilizados para quantificar além da FCmáx, o limiar anaeróbico e o consumo máximo de oxigênio [29]. Dados indicam que existe uma relação forte entre o consumo máximo de oxigênio e a FCmáx, indicando que esse parâmetro pode ser um importante marcador para ganhos de aptidão física [30]. Grande maioria dos estudos utilizou protocolos incrementais, enquanto apenas o estudo de Colantonio e Kiss utilizou o protocolo de Bruce adaptado [22]. De acordo com as diretrizes da American College of Sports Medicine, os protocolos incrementais são recomendados para a avaliação da aptidão física e da FCmáx, levando em conta que se baseia em velocidades ou inclinações crescentes durante todo o teste de exercício [22].

Uma das limitações do presente estudo se caracteriza pela inclusão de um estudo pediátrico obeso nos nossos resultados [16]. No entanto, acreditase que isso não comprometa os achados obtidos, considerando que as outras pesquisas não relataram o estado nutricional de suas amostras, podendo conter sujeitos com sobrepeso e obesidade [1,22,23]. Além disso, todos os estudos incluídos foram classificados com alta qualidade metodológica, o que de certo modo, não inviabiliza os resultados encontrados. Outra possível limitação se trata do fato de não termos testado a validade das equações preditivas na população pediátrica, já que os artigos não apresentaram a análise do coeficiente de correlação intraclasse. Além do mais, o pequeno número de estudos incluídos em nosso trabalho pode constituir-se mais uma limitação dessa revisão.

Os achados da presente revisão sugerem que a equação " $208-(0,7 \times$ idade $)$ " foi a fórmula mais testada e adequada em grande parte para predizer os resultados da FCmáx na amostra pediátrica não atlética. No entanto, nossos achados devem ser interpretados com cuidado, devido a uma pesquisa ter superestimado os resultados com a aplicação dessa fórmula.

Também, parece-nos claro que a estimativa dessa variável não deva ser realizada pela fórmula " 220 idade" devido a superestimativas nas predições realizadas. Mais estudos devem testar a aplicabilidade das equações preditivas e/ou gerar equações baseadas em amostras infantis, levando em conta além da faixa etária, os distintos aspectos amostrais (gênero, atividade física e o estado nutricional).

\section{NOTAS}

Apoio financeiro

Este estudo não recebeu apoio financeiro de fontes externas.

Declaração de conflito de interesses

Os autores declaram não haver conflitos de interesses relevantes ao conteúdo deste estudo.

Contribuições dos autores

Todos os autores fizeram contribuições substanciais para concepção, ou delineamento, ou aquisição, ou análise ou interpretação de dados; e redação do trabalho ou revisão crítica; e aprovação final da versão para publicação.

Disponibilidade dos dados e responsabilidade pelos resultados

Todos os autores declaram ter tido total acesso aos dados obtidos e assumem completa responsabilidade pela integridade destes resultados.

\section{REFERÊNCIAS}

1. Machado FA, Denadai BS. Validade das equações preditivas da frequência cardíaca máxima para crianças e adolescentes. Arq Bras Cardiol. 2011;97(2):136-40. http://dx.doi.org/10.1590/S0066-782X2011005000078

2. Ribeiro VB, de Menezes Reis R, Kogure GS, Tourinho Filho H. Variabilidade da frequência cardíaca em atletas e não atletas saudáveis-diferenças e alterações provocadas pelo treinamento físico de endurance. RBPFEX. 2015;9(54): 446-56.

3. Lopes PFF, Oliveira MIB, André SMS, Nascimento DLA, Silva CSS, Rebouças GM, Felipe TR, Albuquerque Filho NJB, Medeiros HJ. Aplicabilidade clínica da variabilidade da frequência cardíaca. Rev Neurocienc. 2013;21(4):600-3. http://dx.doi.org/10.4181/RNC.2013.21.870.4p

4. Takazono PS, Golin MO. Asfixia perinatal: repercussões neurológicas e detecção precoce. Rev Neurocienc. 2013;21(1):108-17. http://dx.doi.org/10.4181/RNC.2013.21.761.10p

5. Amedro P, Picot MC, Moniotte S, Dorka R, Bertet H, Guillaumont S, Barrea C, Vincenti M, Villeon GL, Bredy C, Soulatges C, Voisin M, Matecki S, Auquier P. Correlation between cardio-pulmonary exercise test variables and health-related quality of life among children with congenital heart diseases. Int J Cardiol. 2016;203:1052-60. http://dx.doi.org/10.1016/j.ijcard.2015.11.028 
6. Melo ALS, Lira YFB, Lima LAB, Vieira FC, Dias AS, Andrade LB. Tolerância ao exercício, função pulmonar, força muscular respiratória e qualidade de vida em crianças e adolescentes com cardiopatia reumática. Rev Paul Pediatr. 2018;36(2):199-206. http://dx.doi.org/10.1590/1984-0462/;2018;36;2;00012

7. Teixeira AL, Moraes EM, Alves HB, Lima JRP. Análise da frequência cardíaca em repouso registrada pré-teste de exercício máximo. Rev Atenção Saúde. 2015;13(45):35-8. https://doi.org/10.13037/ras.vol13n45.2958

8. Condessa LA, Mazini Filho ML, Aidar FJ, Sousa AV, Moura CE, Klain I, Silva AC, Matos DG. Frequência cardíaca máxima: Importância e problemas relacionados a sua medida e estimativa: uma revisão. RBPFEX. 2014;8(47): 428-34.

9. Paschoal MA. Fisioterapia cardiovascular: avaliação e conduta na reabilitação cardíaca. Barueri: Manole; 2010.

10. Gelbart M, Ziv-Baran T, Williams CA, Yarom Y, Dubnov-Raz G. Prediction of maximal heart rate in children and adolescents. Clin J Sport Med. 2017;27(2):139-44. https://doi.org/10.1097/JSM.0000000000000315

11. Meneghelo RS, Araújo CGS, Stein R, Mastrocolla L, Albuquerque P, Serra S. III Diretrizes da Sociedade Brasileira de Cardiologia sobre teste ergométrico. Arq Bras Cardiol. 2010;95(5 supl 1):1-26. http://dx.doi.org/10.1590/S0066782X2010000800001

12. Camarda SR, Tebexreni AS, Páfaro CN, Sasai FB, Tambeiro VL, Juliano Y, Barros Neto TL. Comparison of maximal heart rate using the prediction equations proposed by Karvonen and Tanaka. Arq Bras Cardiol. 2008;91(5):311-4. https://doi.org/10.1590/s0066-782x2008001700005

13. Araújo CGS, Pinto VLM. Frequência cardíaca máxima em testes de exercício em esteira rolante e em cicloergômetro de membros inferiores. Arq Bras Cardiol. 2005;85(1):45-50. http://dx.doi.org/10.1590/S0066-782X 2005001400009

14. Balassiano DH, Araújo CGS. Frequência cardíaca máxima: influência da experiência desportiva na infância e adolescência. Arq Bras Cardiol. 2013;100(4):333-8. http://dx.doi.org/10.5935/abc.20130055

15. Policarpo FB, Fernandes Filho J. Usar ou não a equação de estimativa (220-idade)? Rev Bras Ciência Mov. 2004;12(3):77-80. http://dx.doi.org/10.18511/rbcm.v12i3.578

16. Heinzmann-Filho JP, Zanatta LB, Vendrusculo FM, Silva JS, Gheller MF, Campos NE, Oliveira MS, Feoli AMP, Gustavo AS, Donadio MVF. Frequência cardíaca máxima medida versus estimada por diferentes equações durante o teste de exercício cardiopulmonar em adolescentes obesos. Rev Paul Pediatr. 2018;36(3):309-14. http://dx.doi. org/10.1590/1984-0462/;2018;36;3;00015

17. Vasconcelos TL. Comparação das respostas de frequência cardíaca máxima através de equações preditivas e teste máximo em laboratório. RBPFEX. 2007;1(2):19-24.

18. Barbalho MSM, de Nóvoa HJD, Amaral JC, Lopes TP, Rodrigues PHL, Pires R. Estudo comparativo da frequência cardíaca máxima e estimada por diferentes equações em jogadores de Futebol profissional. RBFF. 2016;8(30):275-9.

19. Caputo EL, da Silva MC, Rombaldi AJ. Comparação da frequência cardíaca máxima obtida por diferentes métodos. Rev Educ Fis UEM. 2012;23(2):277-84. http://dx.doi.org/10.4025/reveducfis.v23i2.12311

20. Roy S, Mccrory J. Validation of maximal heart rate prediction equations based on sex and physical activity status. Int J Exerc Sci. 2015;8(4):318-30.

21. West S, King V, Carey TS, Lohr KN, McKoy N, Sutton SF, Lux L. Systems to rate the strength of scientific evidence. Evid Rep Technol Assess (Summ). 2002;47:1-11.

22. Colantonio E, Kiss MAPDM. Is the HRmax=220-age equation valid to prescribe exercise training in children? JEP. 2013;16(1):19-28.

23. Mahon AD, Marjerrison AD, Lee JD, Woodruff ME, Hanna LE. Evaluating the prediction of maximal heart rate in children and adolescents. Res Q Exerc Sport. 2010;81(4):466-71. http://dx.doi.org/10.1080/02701367.2010.10599707

24. Tanaka H, Monahan KD, Seals DR. Age-predicted maximal heart rate revisited. J Am Coll Cardiol. 2001;37(1):153-6. http://dx.doi.org/10.1016/s0735-1097(00)01054-8

25. Povea CE, Cabrera A. Practical usefulness of heart rate monitoring in physical exercise. Rev Col Cardiol. 2018;25(3):e9-e13. http://dx.doi.org/10.1016/j.rccar.2018.05.004

26. Robergs RA, Landwehr R. The surprising history of the" HRmax=220-age" equation. JEP Online. 2002;5(2):1-10. 
27. Pedroni AS, Schiavo A, Macedo Ed, Campos NE, Winck AD, Heinzmann-Filho JP. Equações preditivas de frequência cardíaca máxima em crianças e adolescentes atletas: uma revisão sistemática. Fisioter Mov. 2018;31: e003131. http://dx.doi.org/10.1590/1980-5918.031.ao31

28. Gellish RL, Goslin BR, Olson RE, McDonald A, Russi GD, Moudgil VK. Longitudinal modeling of the relationship between age and maximal heart rate. Med Sci Sports Exerc. 2007;39(5):822-9. http://dx.doi.org/10.1097/ mss.0b013e31803349c6

29. Herdy AH, Uhlendorf D. Valores de referência para o teste cardiopulmonar para homens e mulheres sedentários e ativos. Arq Bras Cardiol. 2011;96(1):54-9. http://dx.doi.org/10.1590/S0066-782X2010005000155

30. Silva DF, Sotero RC, Simões HG, Machado FA. Velocidad aeróbica máxima calculada por el costo del ritmo cardíaco: relación con el rendimiento. Rev Andal Med Deporte. 2015;8(1):7-15. http://dx.doi.org/10.1016/j.ramd. 2014.06.001 